\title{
Experiments in Carangiform Robotic Fish Locomotion
}

\author{
Richard Mason and Joel W. Burdick \\ \{mason,jwb\}@robotics.caltech.edu \\ Mechanical Engineering, California Institute of Technology \\ Mail Code 104-44, Pasadena, CA 91125
}

\begin{abstract}
This paper studies a form of robotic fish movement that is analogous to the Carangiform style of swimming seen in nature. We propose a simple quasisteady fluid flow model for predicting the thrust generated by the flapping tail. We then describe an experimental system, consisting of a three-link robot, that has been constructed in order to study carangiform-like swimming. Experimental results obtained with this system suggest that the simplified propulsion model is reasonably accurate. The input parameters that realize optimum thmust are experimentally determined. Finally, we consider some issues in manuevering.
\end{abstract}

\section{Motivation}

This paper investigates fish-like robots that propel themselves by changes in their shape, rather than by the use of propellers and maneuvering surfaces. Many fish and marine mammals are impressive swimmers, and fishlike robotic swimmers might be superior to conventional man-made water vehicles in many ways. For decades, biologists and fluid mechanicians have tried to better understand how fish swim so effectively $[1,2,3]$. More recently, interest has grown in developing mechanical imitations of fish, with the idea that such vehicles would be more efficient, more stealthy, and/or more maneuverable than propeller-driven craft. Perhaps the best-known actual robotic fish was built by Triantafyllou et al. [5]. Their highly sophisticated robot represented a fairly faithful reproduction of a swimming tuna. They found that by undulating its highly segmented tail and body, the mechanical tuna was able to reduce the drag it experienced while being towed at constant velocity through the water. This evidence tends to bolster the notion that fish swimming is efficient. It is still an open question whether robot fish can really outperform efficient propeller designs, which themselves have received much research and optimization. The prospect of increased efficiency remains tantalizing, since it would allow battery operated submersibles to operate for longer periods.
Robot fish may be quite stealthy since they do not suffer from the cavitation noise generated by propellers. From a biological standpoint, there is good reason to suppose that fish have evolved to swim stealthily. Ahlborn et al. $[6,7]$ observed that the alternating creation and destruction of vortices in the wake behind the fish was not only an efficient way to swim, but also helped guard against detection by predators. They also built an artificial fishtail to study these effects but their experimental apparatus was not designed to move forward through the fluid, and thus simulated a fish starting from rest rather than continuously swimming.

Furthermore, many fish are highly maneuverable. Some fish can perform a 180 degree turn within a fraction of a body length [8]. This is generally not possible for boats, which typically have large turning radii. Fish are also capable of accelerating very quickly from rest (the escape response) [9]. We suspect that improved agility may ultimately be the biggest advantage that robot fish enjoy over their propeller-driven rivals. Such agility would allow them to work in complex hydrodynamic environments, such as surf surge zones, that are beyond the capability of propeller driven vehicles.

Our work differs from previous studies in two main respects: our experimental apparatus, and our emphasis on modelling for control and motion planning. Our "fish" has a simple construction, consisting of only three rigid links. While this simplicity may limit our ability to imitate biological fish, we hope that it will make the system more tractable for analysis. Our fish is able to propel itself forward without being towed, and we are able to study continuous self-propelled forward swimming. Furthermore, our fish has degrees of freedom enabling it to turn or sideslip as well as swim forward; we have begun work studying issues in fishlike maneuvering. In a previous paper [10] we discussed an earlier version of the apparatus, which was however extremely underpowered and not capable of sideways or turning motions. While possibly interesting as a study of ultra-low-power propulsion, those results 
were of reduced relevance to biological swimming-for example, the Strouhal number of the system was too high compared to natural fishes. We believe the current incarnation of our experiment is much closer to biological fish in a fluid mechanical sense, and perhaps also closer to application in a practical vehicle. More importantly, our system is highly instrumented. With this instrumentation, we are in a unique position to accurately compare theory and experiment, with the result that we can effectively determine what fluid mechanical effects are most important in developing mechanical propulsion models. Prior efforts, such as the simulations spring-driven oscillating-foil vehicles by Harper et al [4] have not been experimentally validated.

Our focus on modelling for control is another somewhat novel aspect of our work. Our long term goal is to develop a rigorous foundation for the design of trajectory generation and feedback control laws that select the patterns of body deformations needed to produce accurate motions that are robust to disturbances. In Ref. [11] we exhibited such a framework for amoeba-like swimmers, where the fluid mechanics is substantially simpler than that which governs carangiform swimming. Past Caltech work has brought the tools of differential geometry to bear on the control of other forms of undulatory locomotion, $[12,13]$ and we hope that these tools can be extended to fishlike swimming. But before we can proceed with this program, we must develop a useful mechanical model of fish locomotion with some experimental validation. This is the topic of this paper.

\section{Review of Carangiform Swimming}

There are a wide variety of fish morphologies and at least a few different types of fish locomotion. We focus on carangiform fishes, fast-swimming fishes which resemble tuna and mackerel. Carangiform fishes typically have large, high-aspect-ratio tails, and they swim using only motions of the rear and tail, while the forward part of the body remains relatively immobile.

To leading order, the geometry of carangiform swimming and the forces related to propulsion can be described as follows. First, we can roughly idealize the main body of the fish as a rigid body. The body is connected to the tail by a penduncle-a slender region of generally negligible hydrodynamic influence. Threedimensional effects are clearly important for many subtle fish maneuevers. However, for purposes of modelling the gross thrust generation process, we simplify the geometry to the two dimensions of the horizontal plane. The 2-dimensional geometry also quite accurately represents our experimental mechanical model.
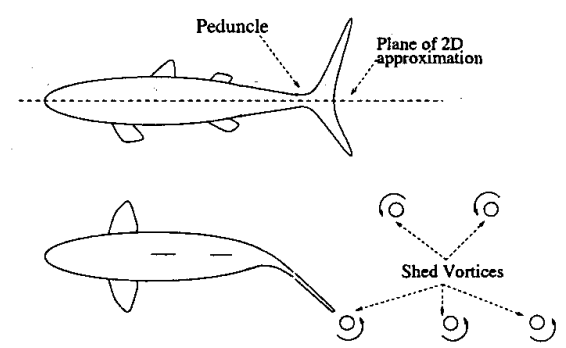

Figure 1: Schematic (side and top view) of Carangiform fish propulsion.

Fish tails are flexible, and this flexibility appears to play an important, but as yet poorly understood, role in propulsion and maneuvering. In our simplified model, the tail can be interpreted as a rigid lifting surface, and its flapping and heaving motions generate thrust in accordance with aerodynamic principles. As the tail moves, non-zero circulation is generated in the surrounding fluid so that the Kutta-Joukowski condition is satisfied. This vorticity is shed into the fish's wake, and the pattern of vortices left behind by the passing of the fish is roughly a reverse Karman vortex street (Fig. 1). This shed vorticity is a source of energy loss [3]. Biological studies [6] suggest that flapping motions of the fish tails are optimized to recapture some of the energy lost in the wake. A vortex is shed near the extremum of the tail's sideways motion. As the tail reverses direction, one can roughly think that the fish "pushes off" of the shed vortex. As a benefit, the vorticity generated by this secondary motion helps to cancel the primary shed vortex, making the wake less detectable by predators. The shed vorticity may also influence the stability of the fish's motion, though there have not yet been significant studies of this effect. Finally, a fluid boundary layer along the fish's body induces drag, and sheds vorticity into the fish's wake. There is sketchy evidence that fish's geometry and tails motions may be adapted to recapture some of this lost energy, thereby improving efficiency.

We conclude this section with a rough sketch of how carangiform swimming is generally understood to work. In Fig. 2, the tail of a fish is shown pitching and heaving up and down as the fish moves from right to left. The basic idea is that the tail maintains a negative angle of attack on the upstroke, and a positive angle of attack on the downstroke, with the result that the lift force on the hydrofoil/tail is always oriented so as to propel the fish forward. Note that in this cartoon, the pitch of the tailfin is zero when the angular excursion of the peduncle is at its maximum, and the pitch of the tailfin is at its maximum when the peduncle is horizontal. In other words, if the tailfin and the peduncle 


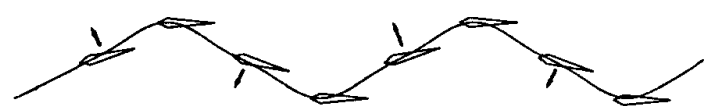

Figure 2: $A$ "cartoon" of carangiform surmming consisting of snapshots of the tail as the fish swims from left to right. The arrows indicate lift forces acting on the tail. The fish body is removed for clarity.

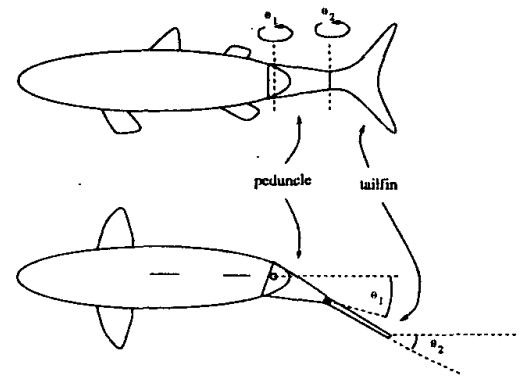

Figure 3: A simplified two-jointed fish model.

motions are considered to be sinusoids, they are ninety degrees out of phase. This amount of phase lag has been reported in biological observations, has been assumed in theory [3], and makes some sense from an intuitive standpoint. Interestingly, we did not find this phase lag to be optimal in our experiment.

\section{An Engineering Thrust Model}

For our model we consider an idealized carangiform fish that consists of only three links: a rigid body in front, a large wing-like tail at the rear, and a slender stem, or peduncle, which connects the two. The three rigid links are connected by rotational joints with joint angles $\theta_{1}$ and $\theta_{2}$. See Fig. 3. We continue to idealize the model by supposing that we can neglect threedimensional effects and regard the problem as essentially planar. In particular, we assume that the large tail can be considered as a rectangular flat plate (although the tails of real carangiform fish are often lunate in shape.) Our experimental apparatus has a flat plate tail. We will presume the tail experiences a hydrodynamic lift force derived from quasi-steady twodimensional wing theory. The peduncle we will regard as hydrodynamically negligible.

Fig. 4 shows the idealized geometry of carangiform swimming obtained by planarization of the $3 \mathrm{D}$ system in Fig. 1. Let $l_{b}$ be the distance between the body's center of mass and the location of the body/peduncle connector. The peduncle has length $l_{p}$. The tailfin has chord $l_{f}$ and area $A$. Let $\vec{l}_{e}$ be a unit vector pointing in the direction of the leading edge of the tailfin hydrofoil. In a coordinate frame aligned with the principal axes

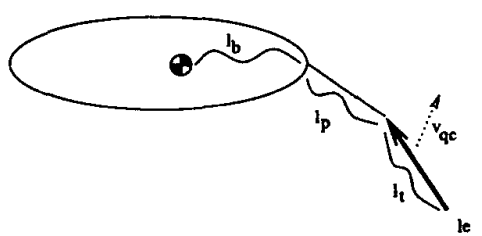

Figure 4: The idealized model.

of the fish's body, then:

$$
\vec{l}_{e}=-\left(\cos \left(\theta_{2}\right), \sin \left(\theta_{2}\right), 0\right)
$$

The body of the fish has instantaneous translational velocity $\dot{x}$ and $\dot{y}$ along its longitudinal and lateral axes; it also has instantaneous rotational velocity $\dot{\phi}$. To apply a standard result in airfoil theory, we need to calculate the velocity of the quarter-chord point, the point at a distance of $1 / 4$ the chord behind the leading edge of the tail. The velocity of the hydrofoil's quarter-chord point, denoted $\vec{v}_{q c}$, is:

$$
\vec{v}_{q c}=\left(\begin{array}{c}
\dot{x}-l_{p}\left(\dot{\phi}+\dot{\theta}_{1}\right) s_{1}-\frac{l_{f}}{4}\left(\dot{\phi}+\dot{\theta}_{2}\right) s_{2} \\
\dot{y}+l_{p}\left(\dot{\phi}+\dot{\theta}_{1}\right) c_{1}+\frac{l_{f}}{4}\left(\dot{\phi}+\dot{\theta}_{2}\right) c_{2} \\
0
\end{array}\right)
$$

where $s_{i}=\sin \left(\theta_{i}\right)$ and $c_{i}=\cos \left(\theta_{i}\right)$. See Fig. 4. Using the Kutta-Joukowski theorem and assuming that the tail hydrofoil is in a quasi-steady uniform flow with the velocity implied by the instantaneous velocity of the foil's quarter-chord point, we arrive at the following lift force on the hydrofoil:

$$
L=\operatorname{sign}\left(\cos \left(\arg \left(\vec{l}_{e}\right)-\arg \left(\vec{v}_{q c}\right)\right)\right) \pi \rho A\left(\vec{v}_{q c} \times \vec{l}_{e}\right) \times \vec{v}_{q c}
$$

The purpose of the first factor in the expression for $L$ is to handle cases when the hydrofoil's angle of attack is between $\pi / 2$ and $3 \pi / 2$ radians, when the trailing edge of the hydrofoil has in fact become the leading edge. The density of the fluid is $\rho$.

In addition to the lift force exerted on the tail, the model fish experiences added mass forces on the body and tail and fluid drag forces on the body which are quadratic in the body's velocity. In the model as implemented, there is also an additional stiction force $f_{s}$ to account for resistance in the carriage bearings which support the experiment. The rigid body has inertia components $I_{x}, I_{y}, I_{\phi}$ (this includes added-mass effects from the fluid surrounding the body) and coefficients of drag $C_{D_{x}}, C_{D_{y}}, C_{D_{\phi}}$. The governing equations of motion are

$$
\left(\begin{array}{c}
I_{x} \ddot{x}_{b} \\
I_{y} \ddot{y}_{b} \\
I_{\phi}
\end{array}\right)=\left(\begin{array}{c}
L_{x}-\frac{1}{2} C_{D_{x}} \dot{x}_{b}\left\|\dot{x}_{b}\right\|-f_{s} \frac{\dot{x}_{b}}{\left\|\dot{x}_{b}\right\|} \\
L_{y}-\frac{1}{2} C_{D_{y}} \dot{y}_{b}\left\|\dot{y}_{b}\right\|-f_{s} \frac{\dot{y}}{\|\dot{y}\|} \\
\tau+\left(x_{m}, y_{m}\right) \times\left(L_{x}, L_{y}\right)-\frac{1}{2} C_{D_{\phi}} \dot{\phi}\|\dot{\phi}\|
\end{array}\right)
$$


Here $\dot{x}_{b}, \ddot{x}_{b}, \dot{y}_{b}$, etc., are the velocities and accelerations of the body expressed in the body-fixed frame, $L_{x}$ and $L_{y}$ represent the components of lift from Eq. 3 , and $\left(x_{m}, y_{m}\right)$ is the location of the midpoint of the tail in that frame. The torque generated around the midpoint of the tail is:

$$
\tau=-\pi \frac{l_{f}^{2}}{4}\left(\dot{x}_{m} y_{m} \cos \left(2 \theta_{2}\right)+\frac{\left(\dot{y}_{m}^{2}-\dot{x}_{m}^{2}\right)}{2} \sin \left(2 \theta_{2}\right)\right)
$$

It is worth stressing how extremely simplified this model is. There are many things not accounted for in the quasi-steady flow model: the after-effect of vortices shed by the body and tailfin ${ }^{1}$; the fundamentally unsteady nature of the flow around the tailfin; the effect of nearby walls and surfaces on the fluid flow (for our experimental fish will swim, not in an unbounded fluid, but in a confined tank.) We have little doubt that any and all of these effects are important in some regimes of the fish's operation, and we anticipate refining our model to include them. However, it is interesting that in the experimental results obtained so far, the simplest model does a surprisingly good job of matching the experimental data.

\section{Description of the Experiment}

We built an experimental "fish" to test the validity of our models. Fig.s 5 and 6 show a schematic diagram of the side and top views of the apparatus, while Fig. 7 shows a photograph of the system. As in the simplified model, the tailfin is a thin flat plate-with a chord of $12.5 \mathrm{~cm}$ and a typical depth in the water of $40 \mathrm{~cm}$. The peduncle is a thin supporting arm, 12.5 $\mathrm{cm}$ in length, which we believe experiences only negligible hydrodynamic forces. The body is a thick flat plate, intended to provide a degree of rotational inertia and rotational damping to help stabilize the fish during planar swimming.

The entire "fish" is suspended in a 4 foot wide by 4 foot tall by 36 foot long water tank from a passive gantry-like multi-degree-of-freedom carriage. The supporting infrastructure consists of two orthogonal sets of rails and a rotating platform, all supported on low friction bearings. By flapping its tail, this mechanism allows the fish to propel itself and its supporting carriage around the tank. The frictional drag on the rails is sufficiently low that this carriage system is a reasonable approximation to untethered swimming. The

\footnotetext{
${ }^{1}$ Note that a large portion of the vortical effects are implicitly included in this model. That is, one can compute from this model the approximate amount of vorticity shed into the wake. However, the shed vortices also have a "backwash" effect on the fish. We do not explicitly account for the influence of this back wash on the bodies' motion or stability.
}

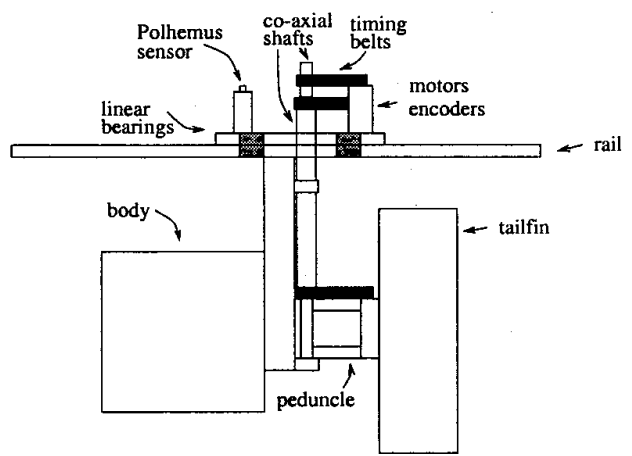

Figure 5: Side view schematic of experiment.

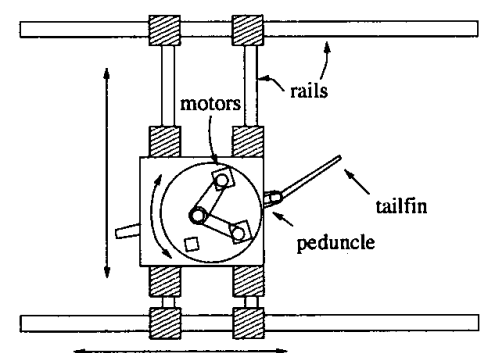

Figure 6: Top view schematic of experiment.

system can move with three degrees of freedom in the plane: forward, sideways, and rotationally. Furthermore, the gantry suspension allows buoyancy effects to be ignored, thereby keeping the experiment focus on thrust generation and maneuvering. The carriage also simplifies the experiment by keeping the motors, electronics, etc. out of the water.

The tail and peduncle degrees of freedom are independently driven by two DC motors (Escap model 35 NT2 R82), each of which is capable of $75 \mathrm{~W}$ of power and $110 \mathrm{mNm}$ of torque. Timing belts and two coaxial shafts transmit power from the motors to the submerged joints of the fish. Also mounted on the carriage are optical shaft encoders (Hewlett-Packard HEDS-5500), which record the fish's joint angles at each instant and enable feedback control of the tail. The encoders are accurate to within about forty minutes of arc. Finally, a Polhemus position/orientation sensor is rigidly attached to the last stage of the carriage. In this way, the absolute position and orientation of the fish can be determined. Since every aspect of the fishes movement is instrumented, we may accurately compare our experimental results with theory for the purposes of assessing the validity of simplified models.

We dragged the passive fish body through the water using a force scale in order to determine the drag and added mass forces acting on the fish in the absence of 


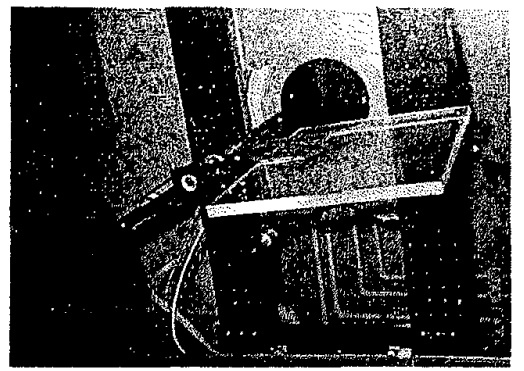

Figure 7: Photograph of mechanical fish from beneath.

any tail movement. The body experienced quadratic drag of about $2 \mathrm{Ns}^{2} / \mathrm{m}^{2}$ in the water, as well as a $2-\mathrm{N}$ stiction force due to the rail bearings.

\section{Experimental Results}

\subsection{Longitudinal Motion/Thrust}

In our initial experiments, the fish apparatus was constrained to move only in a longitudinal direction, with the lateral and yaw degrees of freedom removed. By doing this we, focused on the thrust production aspect of swimming. This procedure also allowed us to find gaits (specific patterns of tail flapping) which produced the maximum forward velocity. An initial investigation of maneuvering is discussed in Section 5.2.

Some experimental results are plotted in Figs. 9 through 13 for several sinusoidal gaits at several frequencies. In each plot, fish position (i.e., distance progressed in the tank) as a function of time is recorded as the fish starts from rest and moves with a specific gait. Each of these plots also compares the actual fish movement to a computer simulation based on the quasi-steady flow model. The computer simulation received as input the actual joint angle trajectories for each gait as reported by the optical shaft encoders. Based on these inputs, the equations of motion developed in Section 3 are integrated, and the simulated fish's displacement is superimposed on the analogous graph of experimental data.

In Fig. 8 we also include some data from an earlier version of the experiment. At that time the fish was underpowered and not capable of more aggressive gaits; also, it did not yet have a "body" link and experienced less drag. In other respects the setup was similar to that used to generate Figs. 9- 13.

Fig. 14 shows the total distance traveled by the fish in an eight second interval after starting from rest. The graph summarizes a large number of sinusoidal gaits, and is plotted as a function of the basic sinusoidal gait parameters: tailfin amplitude and phase. This graph rovides a rough measure of each gait's effectiveness,

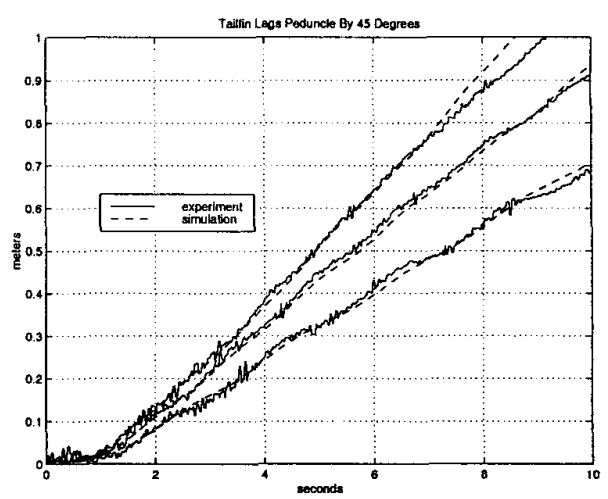

Figure 8: Distance traveled by a low-powered fish vs. time, for gaits of the form $\theta_{1}=0.7 \cos (\omega t)$ and $\theta_{2}=0.9 \cos (\omega t+$ $\pi / 4)$.

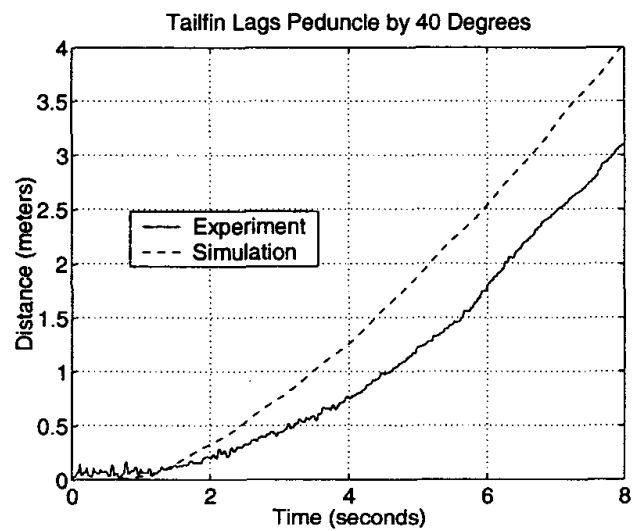

Figure 9: Distance traveled vs. time, for gaits of the form $\theta_{1}=1.3 \sin (3.5 t)$ and $\theta_{2}=0.9 \sin (3.5 t+0.7)$.

and can be used to pinpoint the optimal thrusting parameters.

The following general observations based on these results. First, the quasi-steady model generally does a good job of matching the experimental data. It is particularly good at low speeds and at steady state. Thus, it does a good job of predicting the motion within a few seconds of startup, the overall motion of low-powered vehicles (Fig. 8), and the steady-state forward velocity. The experimental data does deviate sometimes from the computer simulation over intermediate distances. While this difference could indicate a need for improvement of the model through explicit consideration of wake vortices, etc., there is also a more prosaic explanation. The rails on which the experimental carriage rides are not perfectly even and the resistance associated with them varies at different points along the tank. We have not attempted to mold our model to the experimental apparatus by incorporating this spatial variance, as this feature of our experiment is not relevant to untethered operation of robot fish. Fi- 


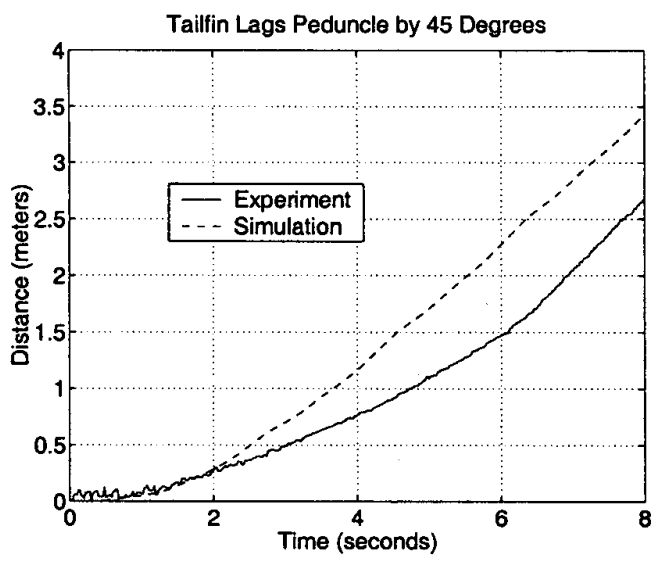

Figure 10: Distance traveled vs. time, for gaits of the form $\theta_{1}=1.3 \sin (3.5 t)$ and $\theta_{2}=1.1 \sin (3.5 t+\pi / 4)$.

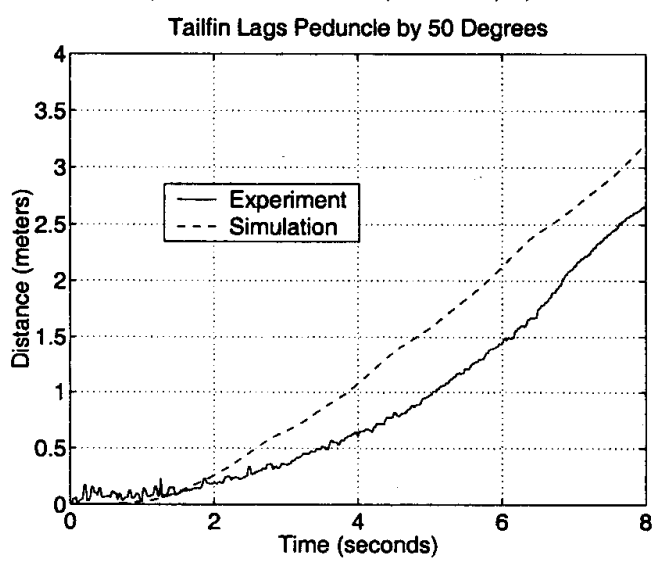

Figure 11: Distance traveled vs. time, for gaits of the form $\theta_{1}=1.3 \sin (3.5 t)$ and $\theta_{2}=0.9 \sin (3.5 t+0.87)$.

nally, note that forward velocity generally increases as tailfin oscillation amplitude decreases. This is consistent with predictions from linear oscillating-foil theory, e.g. Lighthill [3] predicts higher thrust (but lower efficiency) with smaller tailfin oscillations.

Optimal Gait Parameters. Contrary to what we might have expected, the fastest sinusoidal gaits found did not have a phase difference of ninety degrees between $\theta_{1}$ and $\theta_{2}$. Data for low-power gaits [10] showed an optimal phase lag of forty-five degrees, over a range of oscillation amplitudes. At higher speeds the optimal phase lag appeared to be at forty-five degrees or lower, with the fastest experimentally recorded gaits having a phase lag of forty degrees.

Broadly speaking, a smaller phase lag leads to higher velocities of the tailfin quarter-chord point when the peduncle is at its midpoint and the tail is presumably generating the most useful thrust. It is easy to see why this could be advantageous. On the other hand, a

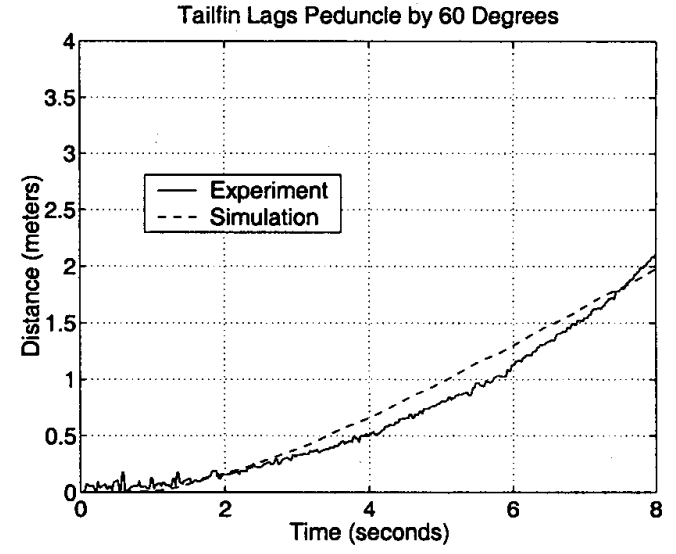

Figure 12: Distance traveled vs. time, for gaits of the form $\theta_{1}=1.3 \sin (3.5 t)$ and $\theta_{2}=0.9 \sin (3.5 t+1.04)$.

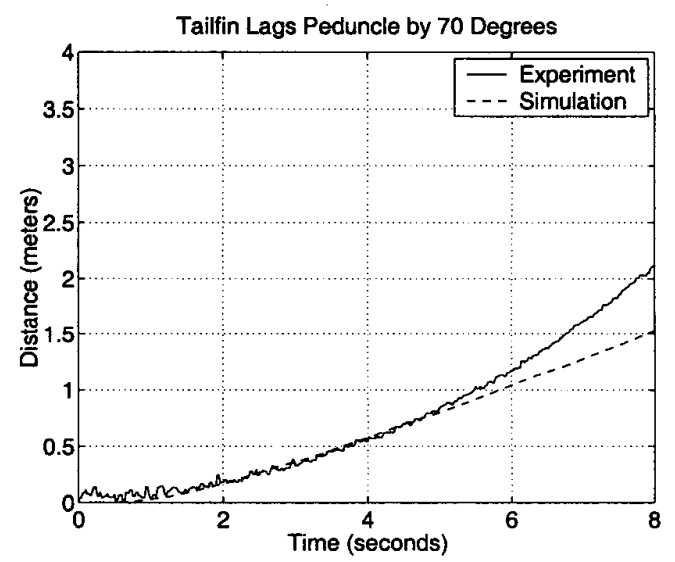

Figure 13: Distance traveled vs. time, for gaits of the form $\theta_{1}=1.3 \sin (3.5 t)$ and $\theta_{2}=0.9 \sin (3.5 t+1.22)$.

small phase lag would seem to create unnecessary drag when the peduncle is at its extremities and no useful forward thrust can be generated. Seemingly the higher thrust of a small phase lag outweighs the higher drag, at least in our experiment, which raises the question of why the same is not observed in biological fish. Possibly the answer is that strictly sinusoidal motions of the tailfin are not an adequate model for the motions of real fish. It is plausible that even better thrust could be generated by a periodic tailfin motion that was not sinusoidal, and therefore could not be described as having a constant phase lag with respect to the sinusoidal peduncle motion. This non-sinusoidal path of the tailfin could be shaped to have good characteristics at both the extremes and the midpoint of the stroke. This is a subject for further research.

Strouhal Number. Triantafyllou et al. [14] em- 


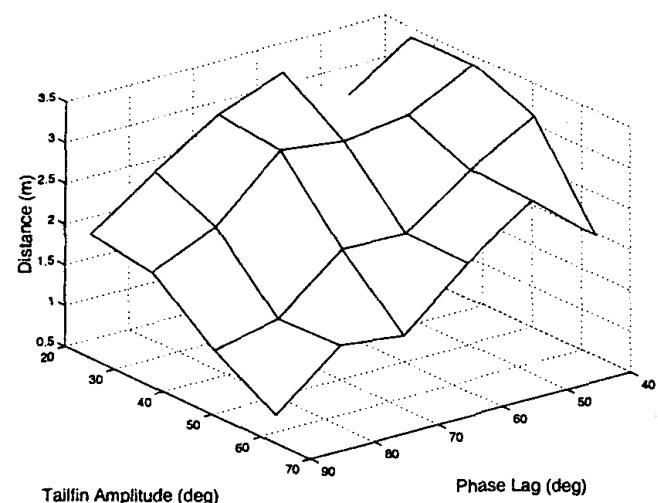

Figure 14: Distance traveled by the fish in eight seconds, for gaits of the form $\theta_{1}=1.3 \sin (3.5 t)$ and $\theta_{2}=A \sin (3.5 t+$ $\psi)$. The horizontal axes represent the parameters $A$ and $\psi$.

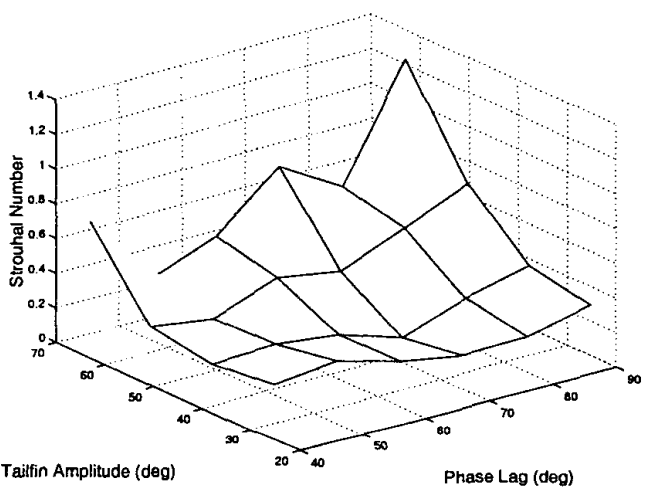

Figure 15: Strouhal numbers, for gaits of the form $\theta_{1}=$ $1.3 \sin (3.5 t)$ and $\theta_{2}=A \sin (3.5 t+\psi)$. The horizontal axes represent the parameters $A$ and $\psi$.

phasized the importance of the dimensionless Strouhal number, defined as $\mathrm{St}=\frac{\mathrm{fA}}{\mathrm{V}}$ where $f$ is the frequency of the tailfin oscillation, $A$ is the double-amplitude of the tail-to-tip excursion, and $V$ is the average forward swimming velocity. Triantafyllou et al. advanced arguments that the Strouhal number of an oscillating foil system should be in the range $0.25-0.35$ for optimum thrust. They also cited biological observations suggesting that a wide range of fish and cetaceans actually do operate in this range. In Fig. 15, we plot the Strouhal number for the same set of sinusoidal gaits represented in Fig. 14. It will be seen that the Strouhal number for our system is precisely in the optimum range, particularly for the fastest gaits. (Note that the horizontal axes in Figures 15 and 14 are reversed, to increase visibility of the surfaces.) This tends to further support Triantafyllou's observations and also suggests that our system is operating in a fluid mechanical regime fairly representative of biological fish swimming.

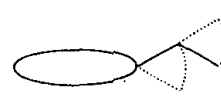

(a.)

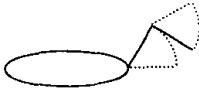

(b.)

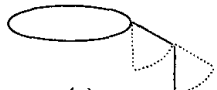

(c.)
Figure 16: (a): Forward swimming. (b) and (c): Two schemes for turning counter-clockwise.

\subsection{Turning Gaits}

After identifying successful gaits for forward swimming, we began to experiment with unconstrained maneuvers such as turns. Turning in some species of fish has been experimentally studied, and has been qualitatively described and understood [8]. However, a useful mathematical model has not yet been put forth. Here we present some basic motion primitives for turning of our carangiform swimmer. These primitives do not necessarily have analogs found in nature. One way to cause the fish to turn or yaw is to carry out a gait similar to forward swimming, but with the peduncle's motion offset by some amount. In this case the average thrust generated will still be in a mostly forward direction, but it will produce a non-zero timeaverage torque around the center of mass. (See Figure 16(b).) Another idea is to rotate the ranges of motion of both the peduncle and the tailfin, thus generating a mostly-lateral thrust and a non-zero torque. (See Figure 16(c).) In both of these schemes, the tailfin is treated more or less as a thruster whose time-averaged thrust vector can simply be redirected. Which of the two schemes is more effective will depend on the desired thrust direction during the turn and also on details of the fish's body and the location of the center of mass. A few turning gaits of type (b) are illustrated in Fig. 17. The variable $\psi$ as usual refers to phase lag between the peduncle and the tailfin.

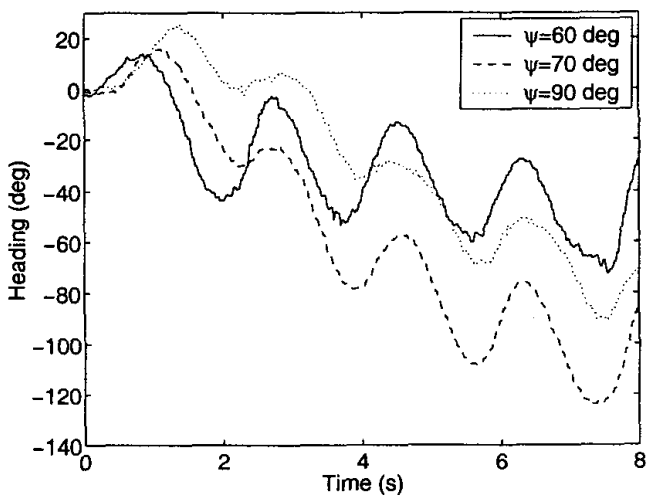

Figure 17: Orientation change over time, for gaits of the form $\theta_{1}=-0.6+1.3 \sin (3.5 t)$ and $\theta_{2}=1.1 \sin (3.5 t+\psi)$.

Changes in the stroke's range of motion should be synchronized to the periodic motion of the tail, so probably the tail should only be re-oriented to a new range 
of motion in between propulsive tail beats. This suggests a planning method. If a variety of strokes are each known, through simulation or experiment, to produce a given impulse and angular impulse per beat, then the fish could choose individual beats as necessary to add a quantized amount of momentum or angular momentum. Maneuvers could then be built up of series of such individual strokes. There seems to be an analogy between these quantized strokes and the "impulse bits" of thrusters used to orient spacecraft.

However, while the modes of turning illustrated in Fig. 16 may be useful for small course adjustments-for example, small corrections necessary to keep the fish on a constant heading-neither of them is entirely satisfactory since a large part of the appeal of a fishlike swimmer must be its ability to perform a very rapid turn in a small space, with a single dramatic body motion. The turning of biological fish has been described by Weihs [8]. Imitating these large turning motions with our apparatus is an area for further research. Biological fish curve their whole spine during such turns, including significant motion of the head, and it remains to be seen if we can adequately imitate these motions using a three-link apparatus.

\section{Future Work}

Carangiform-like swimming holds much promise for robotic fish. However, accurate and efficient robotic swimming will ultimately require precise models for the mechanics that govern such motions. This paper took some initial steps to fill this gap. We introduced a simplified model for carangiform swimming based on quasi-steady flow theory. Using a novel instrumented experimental carangiform swimmer, we showed that this model is reasonably accurate and useful for modelling thrust generation. Additional work is clearly needed to incorporate and experimentally investigate the influence of the other non-steady and nonlinear fluid effects that are not included in the present model. In particular, the advanced models must predict the fish's lateral and yaw stability properties. A greater understanding of rapid turning maneuvers must be realized. Ultimately, these types of results would form the basis for further work in trajectory generation and active feedback control. In particular, we intend to explore feedback control laws that take the velocity of the fish as an input. Truly optimal fish locomotion is likely to involve tuning the angle of attack of the tailfin at each instant, which in turn requires incorporating feedback about the fluid flow around the fish into the control scheme. This suggests that entirely new sensing technologies are required.

\section{References}

[1] S. Childress. Mechanics of Swimiing and Flying. Cambridge University Press, Cambridge, 1981.

[2] J. N. Newman and T. Y. Wu. Hydrodynamical aspects of fish swimming. In T. Wu, C. Brokaw, and C. Brennen, editors, Swimming and Flying in Nature, Vol 2., pages 615-634. Plenum Press, New York, 1975.

[3] J.L. Lighthill. Mathematical Biofluiddynamics. Society for Industrial and Applied Mathematics, Philadelphia, 1975.

[4] K. A. Harper, M. D. Berkemeier, and S. Grace. Modeling the dynamics of spring-driven oscillatingfoil propulsion. IEEE J Oceanic Eng., 23:(3):285-296, 1998.

[5] J. M. Anderson, K. Streitlien, D. S. Barrett, and M. S. Triantafyllou. Oscillating foils of high propulsive efficiency. J Fluid Mech., 360:41-72, 1998.

[6] B. Ahlborn, D. G. Harper, R. W. Blake, D. Ahlborn, and M. Cam. Fish without footprints. J Theor Biol., 148:521-533, 1991.

[7] B. Ahlborn, S. Chapman, R. Stafford, R. W. Blake, and D. G. Harper. Experimental simulation of the thrust phases of fast-start swimming of fish. $J$ Exper Biol., 200:2301-2312, 1997.

[8] D. Weihs. A hydrodynamical analysis of fish turning manoeuvres. Proc. R. Soc. Lond., B. 182:59-72, 1972.

[9] D. Weihs. The mechanism of rapid starting of slender fish. Biorheology, 10:343-350, 1973.

[10] R. Mason and J. Burdick. Construction and modelling of a carangiform robotic fish. In International Symposium on Experimental Robotics, Sydney, Australia, 1999.

[11] R. Mason and J. Burdick. Propulsion and control of deformable bodies in an ideal fluid. In IEEE International Conference on Robotics and Automation, Detroit, 1999.

[12] J.P. Ostrowski. The Mechanics and Control of Undulatory Robotic Locomotion. PhD thesis, California Institute of Technology, Pasadena, CA, Sept. 1995.

[13] S. Kelly and R. Murray. Geometric phases and robotic locomotion. J. Robotic Systems, 12(6):417-431, June 1995.

[14] G. S. Triantafyllou, M. S. Triantafyllou, and M. A. Grosenbaugh. Optimal thrust development in oscillating foils with application to fish propulsion. J Fluid Struct., 7:205-224, 1993.

[15] D. Weihs. Some hydrodynamical aspects of fish schooling. In $\mathrm{T}$. $\mathrm{Wu}, \mathrm{C}$. Brokaw, and $\mathrm{C}$. Brennen, editors, Swimming and Flying in Nature, pages 703-718. Plenum Press, New York, 1975. 